\title{
Extensively drug resistant tuberculosis
}

\author{
A serious wake-up call for global health
}

$\mathrm{T}$ Tuberculosis outbreaks in the developed world are newsworthy. ${ }^{1}$ However, in the developing world, where deaths from tuberculosis are common, it takes something exceptional for an outbreak to attract much attention. In response to a recent report at the 16th international AIDS conference $^{2}$ and to increasing South African media reports, the World Health Organization last week expressed concern about extensively drug resistant tuberculosis (also referred to as "XDR tuberculosis")."

Among 536 culture confirmed cases of tuberculosis at a rural hospital in South Africa, $41 \%$ were multidrug resistant, ${ }^{2}$ defined as resistance to rifampicin and isoniazid (two key first line drugs). This is cause enough for concern as multidrug resistant tuberculosis has a worse outcome and its management is very difficult even in high resource settings. ${ }^{4}$ Even more alarming was that $53(24 \%)$ of the isolates from multidrug resistant tuberculosis fulfilled the definition of extensively drug resistant tuberculosis ${ }^{2}$-namely, multidrug resistant tuberculosis that is also resistant to at least three of the six classes of second line agents. Such tuberculosis is virtually untreatable.

All patients in this outbreak who were tested were HIV infected, and 52 of the 53 died after a median of just 25 days. $^{2}$ In $90 \%$ of the isolates the same genetic fingerprint was present, indicating extensive recent transmission. Fifty six per cent of patients had previously been admitted to hospital, raising the likelihood of nosocomial transmission.

Outbreaks of infectious diseases are always more newsworthy if their implications extend beyond the local context, which is the case with extensively drug resistant tuberculosis. For some years, such strains have been known to exist in Asia, North and South America, and Europe. In March this year, the Centers for Disease Control and Prevention and WHO reported a survey of over 17000 tuberculosis isolates collected from around the world between 2000 and 2004. ${ }^{5}$ Overall, 2\% of multidrug resistant strains were also extensively drug resistant, being most frequently found in eastern Europe, western Asia, and South Korea. Population based data from the United States, Latvia, and South Korea showed that $4 \%, 19 \%$, and $15 \%$ respectively of multidrug resistant strains could be defined as extensively drug resistant.

The epidemiology and the limited genotypic data currently available ${ }^{26}$ indicate that this is not a single strain, but that extensively drug resistant strains are likely to have emerged in many different places and on multiple occasions. Paradoxically, this is both reassuring and alarming. It is reassuring in that the emergence of extensively drug resistant tuberculosis in more than one strain suggests that the mutations responsible are specific for drug resistance rather than reflecting a fundamental change in behaviour of the organism. This is nevertheless alarming because it also suggests that extensively drug resistant tuberculosis probably arises fairly regularly and is already disseminated.

Drug resistance to tuberculosis results largely from poorly managed care and control of the disease. Poor prescribing practices, low drug quality (or erratic supply), and suboptimal adherence can all contribute to this. Bacilli are subject to intense drug selection, and exposure to monotherapy predisposes to an accumulation of mutations that confer resistance. Hence optimal treatment includes four drugs to which the organism is sensitive, and a single drug should never be added to a failing regimen. In much of the world, routine culture and sensitivity testing is not available. Thus, where multidrug resistant tuberculosis emerges, inappropriate treatment regimens may lead to serial acquisition of resistance mutations, with potential for emergence of extensively drug resistant tuberculosis. Widespread use of second line tuberculosis drugs (such as quinolones for respiratory tract infections) may also contribute to the development of resistance. Thus, the emergence of extensively drug resistant tuberculosis should come as no surprise-it was entirely predictable in the context of poor control practices.

The havoc that institutional transmission of multidrug resistant tuberculosis can wreak amongst HIV infected people was evident in the US in the early 1990s. ${ }^{7}$ The very modest actual rise in the incidence of tuberculosis that coincided with these outbreaks has now been reversed, ${ }^{8}$ albeit with extraordinary effort and cost. However, the huge potential for extensively drug resistant tuberculosis to further undermine control practices in communities in South Africa and elsewhere in the region is self evident and would be much more difficult to control. In some communities with an antenatal prevalence of $\mathrm{HIV}$ of $30 \%$, annual notification rates for tuberculosis have already increased uncontrollably over the past 10 years, reaching 1500/100 000-a rate more than 250 times higher than rates in the US. ${ }^{9}$ Extensively drug resistant tuberculosis must now serve as a serious wake-up call. Although the potential consequences may be most grave in settings with a high prevalence of tuberculosis and HIV, extensively drug resistant tuberculosis is nevertheless already a very serious development in many other parts of the world too. ${ }^{5}$ 
What response is needed? The global scale and molecular epidemiology of extensively drug resistant tuberculosis require urgent assessment, and laboratory capacity needs to be greatly increased within a network of sentinel sites. Control practices must be rigorously and effectively implemented. Increasing cure rates for tuberculosis through directly observed treatment short course (DOTS) is crucial. Detection rates for cases of tuberculosis need to be improved, highlighting the need for a new diagnostic test. Technologies that can determine the presence of drug resistance at the point of care are needed, as are new drug treatments. The DOTS-Plus strategy ${ }^{10}$ for treatment of multidrug resistant tuberculosis needs to be further developed for areas where the disease is established. Nosocomial transmission of tuberculosis is probably commonplace in the developing world, and simple, effective strategies

1 Watson JM, Moss F. TB in Leicester: out of control, or just one of those things? BMJ 2001;322:1133-4.

2 Ghandi NR, Moll A, Pawinski R, Sturm AW, Lalloo U, Zeller K, et al. High prevalence and mortality from extensively-drug resistant (XDR) TB in TB/HIV coinfected patients in rural South Africa. Abstracts of the 16th international AIDS conference, 13-18 August 2006. Toronto, Canada: International AIDS Society, 2006. (Abstract THLB0210.)

3 World Health Organisation. Emergence of XDR-TB. WHO concern over extensive drug resistant TB strains that are virtually untreatable. www.who.int/mediacentre/news/notes/2006/np23/en/index.html (accessed 10 Sep 2006).

4 Mukherjee JS, Rich ML, Socci AR, Joseph JK, Viru FA, Shin SS, et al. Programmes and principles in treatment of multidrug-resistant tuberculosis. Lancet 2004:363:474-81.

5 Emergence of Mycobacterium tuberculosis with extensive resistance to second line drugs worldwide, 2000-2004. MMWR Morb Mortal Wkly Rep 2006;55:301-5 to reduce such transmission need to be urgently implemented. More fundamentally, the emergence of extensively drug resistant tuberculosis is a reminder that tuberculosis needs massive broader commitment: the incompletely funded Global Plan to Stop TB $^{11}$ demands political will and financial action.

Stephen D Lawn senior lecturer in infectious and tropical diseases

(stevelawn@yahoo.co.uk)

Desmond Tutu HIV Centre, Institute of Infectious Disease and Molecular Medicine, Faculty of Health Sciences, University of Cape Town, Anzio Road, Observatory 7925, Cape Town, South Africa

Robert Wilkinson Wellcome Trust senior fellow in clinical tropical medicine

Institute of Infectious Disease and Molecular Medicine, University of Cape Town

Competing interests: None declared.

6 Masjedi MR, Farnia P, Sorooch S, Pooramiri MV, Mansoori SD, Zarifi AZ, et al. Extensively drug-resistant tuberculosis: 2 years of surveillance in Iran. Clin Infect Dis 2006;43:841-7.

7 Frieden T, Sterling T, Pablos-Mendez A, Kilburn J, Cauthen G, Dooley S. The emergence of drug-resistant tuberculosis in New York City. $N$ EnglJ Med 1993;328:521-6.

8 Trends in tuberculosis-United States, 1998-2003. MMWR Morb Mortal Wkly Rep 2004;53:209-14.

9 Lawn SD, Bekker LG, Middelkoop K, Myer L, Wood R. Impact of HIV on epidemiology of tuberculosis in a peri-urban community in South Africa: epidemiology of tuberculosis in a peri-urban community in South Afric:
the need for age-specific interventions. Clin Infect Dis 2006;42: 1040-7.

the need for age-specific interventions. Clin Infect Dis 2006;42: 1040-7.
0 Farmer P, Kim JY. Community based approaches to the control of multidrug resistant tuberculosis: introducing "DOTS-plus." BMJ 1998;317:671-4.

11 Stop TB partnership. The global plan to stop TB 2006-2015. Executive summary. 2006. www.stoptb.org/globalplan/assets/documents/GP_ES_ Eng.pdf (accessed 10 Sep 2006).

doi 10.1136/bmj.38971.587222.AB

\section{Emergency contraception}

\section{Is it worth all the fuss?}

$\mathrm{E}$ mergency contraception can prevent pregnancy after unprotected sex, but it can also cost you your job. In 2005 an assistant commissioner resigned from the Food and Drug Administration (FDA) in the United States after a decision to make emergency contraception available off prescription was postponed indefinitely, despite two committees recommending it (after three years' delay the FDA has recently approved over the counter sales, with restrictions, of the emergency contraceptive Plan B). ${ }^{1}$ In 2006 two editors of the Canadian Medical Association Journal (CMAJ) were fired, partly because they published an article about access to emergency contraception in Canadian pharmacies. ${ }^{2}$ Emergency contraception has been described as "the latest battleground in an ideologically divided America." It has always been a battleground, but is it worth all the fuss?

First used in the early 1970s, emergency contraception was a well kept secret until the late 1990s. At this time interest in this form of contraception exploded and considerable efforts were made to promote it. Dedicated products are now available in many countries, increasingly off prescription. Its use in most countries is low, however. A minor proportion of women undergoing abortion claim to have used emergency contraception in the past to try to prevent preg- nancy $\left(1.3 \%\right.$ in the US, ${ }^{4} 2.9 \%$ in Sweden, and 9.2\% in France). In the United Kingdom its use has grown from $1 \%$ among women requesting an abortion in 1984, to $6 \%$ in $1996,{ }^{5}$ and $12 \%$ in $2002 .{ }^{6}$

Emergency contraception has been heralded as the solution to rising abortion rates. Some authors have suggested that almost a million abortions could be prevented in the US annually if every woman used emergency contraception every time she needed it. ${ }^{7}$ Proponents claim that $43 \%$ of the reported fall in abortions in the US (110 000 between 1994 and 2000) was due to use of emergency contraception, and that around 51000 pregnancies were prevented by its use in 2000-1. ${ }^{4}$ Similar calculations would lead us to conclude that emergency contraception prevented more than 66500 abortions in England and Wales in 2004.

Yet, despite the clear increase in the use of emergency contraception, abortion rates have not fallen in the UK. They have risen from 11 per 1000 women aged 15-44 in 1984 (136 388 abortions) to 17.8 per 1000 in 2004 (185400 abortions). Similarly, increased use of emergency contraception in Sweden has not been associated with a reduction in abortion rates. $^{8}$ A multitude of social and economic factors influence pregnancy rates, and it is hard to show the effect of a single factor. For example, the fall in the 